\title{
On Language Evolution
}

\author{
José-Luis Mendívil-Giró
}

In response to "Misused Terms in Linguistics" (Vol. 5, No. 2).

To the editors:

I share Evelina Leivada's conclusion that terminological clarity matters. Leivada has done a great job compiling in a few pages the overwhelming issue of harmful vagueness in linguistics terminology. Her selection is relevant and ambitious. In this letter, I would like to add another expression that in my opinion is frequently misused in current linguistics: language evolution.

In English, the term "language evolution" presents an "unfortunate ambiguity," to use James Hurford's term, due to the fact that, unlike what happens in French and other languages, the same word is used to designate the languages spoken by people (French langue) and the capacity of language as such (French langage). Language evolution refers, in one of the senses, to the evolution of language as a characteristic faculty of the human species and, in the other sense, to the fact that languages change over time. Typically, the context is sufficient to distinguish between both uses. In current linguistics, things get complicated because a strong tradition has resurfaced according to which both processes are closely connected. Indeed, for many authors, ${ }^{2}$ language evolution is a consequence of language change, so the two phenomena are mixed and even identified as the same.

Sticking with the traditional use of the terms, I argue here that the term "language evolution" should be used to refer to the plausibly biological processes that formed the modern human faculty of language, and that the term "language change" should be reserved to refer to processes that alter the structure of languages over historical time, as in, for example, the shift from Latin to Spanish. With this proposal, I take sides with those authors for whom there is no connection between the fact that languages change over time and the fact that the human faculty of language is the result of natural evolution. ${ }^{3}$ But even if I fail to convince the reader that language evolution cannot be explained as a consequence of language change, I think it is necessary to maintain this terminological distinction. ${ }^{4}$

\section{Language Evolution and Language Change}

Language change is a cultural phenomenon that occurs on the scale of historical time, over hundreds or thousands of years. Its main consequence is the creation of new languages through the modification of others, just as Spanish emerged from Latin some 1,500 years ago, or just as Latin emerged from Proto-Indo-European around 5,000 years ago.

In contrast, language evolution is not a cultural process, but is part of natural evolution, which occurs on a geological timescale of hundreds of thousands and millions of years. Its main consequence is the appearance of the human capacity for language sometime between 6 million and 100,000 years ago.

There is, of course, no reason not to use the word "evolution" to refer to cultural changes, and, therefore, to historical change in languages. In fact, there are some signs of evolution in language change. ${ }^{5}$ But my argument is that interchanging both types of evolution-language change and natural evolution-is misleading and empirically inadequate. Reserving the term "evolution" for biology is an arbitrary convention, but a necessary one to avoid confusing two phenomena of very different natures. As Noam Chomsky has suggested,

Confusion about these matters could be mitigated by replacing the metaphorical notions "evolution of language" and "language change" by their more exact counterparts: evolution of the organisms that use language, and change in the ways they do so. In these terms, emergence of the language faculty involved evolution, while historical change (which goes on constantly) does not. ${ }^{6}$

The main reason to combat vagueness in the use of the expression "language evolution" is that it is not possible to explain the origin of human language as a process of cultural change from the languages of the ancestors of Homo sapiens. Cultural evolution involving phonetic, syntactic, and semantic changes in the ways of speaking is insufficient to explain the change in the language capacity of human beings with respect to their closest living evolu- 
tionary relatives, the chimpanzees, which separated from our common evolutionary trunk some 6 million years ago . It also took a very intense biological evolution in the anatomy and physiology of the brain, at least.

Our language is not only a cultural invention, but also a specifically human cognitive system, including all those cognitive developments that we may have shared with our most immediate extinct ancestors. The languages spoken by human beings are not languages that have changed, or evolved, from chimpanzee languages or from the primitive languages of other species. They are instead based on human cognitive attributes that are the result of the biological evolution of our species. Thus, the differences between language change and language evolution have to do with the different nature of the objects that change in each case: human languages and the faculty of language.

\section{What Language Is and What Languages Are}

Human language is a system of knowledge, a cognitive organ, common to all members of the species: all people learn a language, except those with severe pathologies. It is also specific to the species: no other organism, natural or artificial, can learn a language. Although there are many different languages, they all belong to the same cognitive class-human language. To put it briefly, languages are different forms, historically modified, of a single cognitive capacity, language.

As Samuel Epstein et al. remark, people are not like tape recorders, capable of recording and reproducing the sounds of the environment, but they are capable of creating and understanding expressions that they have never heard or produced before. ${ }^{7}$ There is something in their nature that makes them capable of developing an unlimited knowledge system from a limited exposure to stimuli from the environment. In addition, the stimuli that children perceive, against intuition, do not contain phonemes, morphemes, words, or sentences, but are simply noises, disturbances of air molecules that press the eardrums, perceived in certain circumstances. ${ }^{8}$

In this context, a crucial question arises: How is it possible for a finite organism to develop the knowledge of entities that are not present in the stimuli of the environment, including digital infinity? The well-known answer Chomsky suggested is that we must assume that a central part of human cognition is a recursive computational system. Actually, his answer is inspired by the same problem that arises in biology to explain the development of organisms: How does a frog develop from a tadpole?

As in the biological sphere, a cognitive organ such as language develops from a genetic program in interaction with environmental stimuli of a certain type. Tadpoles do not become frogs because of the type of food they receivethere is no frogness in their food. Inadequate food intake can ensure that the frog does not develop well, but not that the tadpole turns into something else, like a salamander. It is the organism itself, its internal program, written by natural evolution, that determines which stimuli are relevant to development. In essence, the same thing happens with ontogenetic language development. Certain external stimuli are required for language to develop according to an internal program; these stimuli do not contain syntax or semantics, but are, in the usual case, certain types of acoustic disturbances.

Nonetheless, there is an important difference: tadpoles always produce frogs, but not all people end up speaking the same language. Note that, although frogs are very similar to each other, they are not identical. Of course, the differences between languages are very notable, as evidenced by the fact that understanding one does not guarantee understanding the others. But this does not mean that they cannot be conceived as relatively superficial variants of the same knowledge system, built by natural evolution.

To better understand the difference between what has biologically evolved-the capacity of language-and what has historically changed-languages-it is useful to differentiate between two different states of a person's faculty of language (FL).

On the one hand, there is the initial state of the FL. The initial state of the FL includes all the biological properties that make it possible for any human being to be able to learn and use any language. Chimpanzees or parrots lack these properties. Raised in an environment similar to that of children, they do not learn Spanish, English, or Japanese. The argument that this deficiency is not due to a lack of a faculty of language but of other general cognitive abilities is strange, if not incoherent. Whatever its origin, there is an innate capacity in human nature that is not present in other organisms. This capacity is the result of biological evolution and constitutes the initial state of the FL.

Children do learn languages, spontaneously and effectively. When children reach puberty, they already have a full knowledge of the grammar of their mother tongue(s). Let's say they have moved from the initial state to the steady state of the FL. Of course, some aspects of their language will continue to change throughout life: new words, new meanings, mastery of special registers, etc. But it will no longer happen that their language becomes another. Borrowing Chomsky's terminology, we can call each person's steady state of FL its internal language (I-language). Each person has at least one I-language in the brain, which is the result of the development of the initial state of FL. An I-language is a person's language organ.

The initial state is, by definition, common to all members of the species and, therefore, universal-invariable in space and time. It is the result of natural evolution and does not change in historical time. The I-language, however, is not common and universal, but, varies in each individ- 
ual, as each frog is different from the others. Of course, a person's I-language does not vary as much as to be very different from the I-languages of the other members of the same community, so we do not say that each person speaks a different language. Instead, we group people's I-languages by their degree of similarity. These groups of similar I-languages are what we informally call languages, such as Spanish, English, or Japanese. What we normally call "a language" is just a group of similar I-languages-or an E-language, with "E" standing for external and extensional. There are billions of I-languages, at least as many as there are people. But they can be grouped into some five or six thousand different E-languages, the number of different languages that are spoken in the world today.

\section{Why There Are Different Languages}

Although human beings are endowed with the same language capacity, not all human beings speak the same language. Why is it that all people start from the same initial state, but not everyone reaches the same steady state? The explanation has to do with the role of environmental stimuli in the development process that leads from the initial to the steady state. Unlike what happens with frogs, or with lungs, or with the visual system, the language organ, in addition to its own development program, has a cultural component that is learned from the environment. This component is susceptible to historical changes in its transmission from generation to generation.

Chomsky's claim that an alien scientist would conclude that all humans speak the same language is as famous as it is controversial. But, as human researchers, we observe that each natural species, whether blue whales, chimpanzees, or bees, has a single communication system, and not several. Each species is equipped with its own communication system that is biologically determined. This implies that individuals do not have to learn it, but it is part of their nature, as are fins, hair, or the instinct to search for pollen. What Chomsky wants to imply, therefore, is that human beings, despite appearances, do not have to be different.

And we are not. There are about six thousand languages in the world-that is, about six thousand different ways of speaking that do not allow mutual understanding. To explain this fact, it is necessary to consider in a little more detail the language of other animals, such as whales or certain songbirds. Ethologists have observed that there are subtle differences in the songs of different groups of whales of the same species and in the songs of different groups of birds of the same species. Some birds do not precisely imitate the singing of adults, but instead produce certain innovations that make the songs they use no longer identical to what they heard when they were nestlings. This causes successive generations of birds to hear slightly different songs than the previous generation, resulting in birds of the same species from different regions singing different songs.

This is not all that different from what happens with human languages: in each of those six thousand different languages there are different dialects. The difference between dialects and languages is not a class difference, but a reflection of the similarity between two different ways of speaking. Two different ways of speaking English are considered two dialects of the same language because these forms are similar enough-their users usually understand each other. The much fewer similarities between a French speaker and an English speaker means that their ways of speaking cannot be considered dialects of the same language. Instead, they are considered different languages, because these forms are much less similar, and their users do not understand each other. Whales or songbirds supposedly only have dialects because the margin of variation in their systems is small, while users of human language not only have dialects, they also have languages because the margin of variation in human language allowed by biology is greater than in non-human language.

The question to answer, then, is why human language allows more variation than the rest. Perhaps the answer has to do with what kind of animals we humans are. We have a language specific to our species, common to all people and different from those of other species. But, on the other hand, we are special animals, in the sense that we are much more capable to learn from the environment and develop and transmit culture. Nature has endowed us with an incredible ability to learn, including the ability to enrich our FL by developing it in interaction with other minds, which has the consequence that we can enjoy languages of a complexity that could not be genetically encoded. Any human language has many more words than there are genes in the human genome, which is about 20,000 .

The consequence of having to learn some aspects of language is that it is not only normal that different dialects arise, but also that they differ so much from each other that they end up giving rise to what we call different languages. There is a correlation between the degree of variation of a system and the degree of learning involved in its development.

\section{What Changes When Languages Change}

Despite what it may seem when we compare Latin and Spanish, linguistic changes do not have the capacity to alter the common internal structure of languages. Changes can only alter their surface. This is precisely why we cannot accept that the evolution of language can be explained as a succession of linguistic changes that would convert supposed primitive languages or prehuman languages into the human languages that we speak now. 
But what is the surface of a language? To answer this question, we must consider which components make up an I-language. According to the influential model proposed by Chomsky, Marc Hauser, and W. Tecumseh Fitch, the human FL includes, minimally, three main components: a conceptual system related to semantic interpretation, a sensorimotor system related to the production and perception of sounds and visual signs, and a computational system, which is the syntax in the narrow sense responsible for the compositional and productive structure that underlies linguistic expressions. ${ }^{9}$

The relevant question now is in which of these three components-semantics, phonetics, or syntax-do the changes that result in the diversity of languages occur. The answer, although it seems surprising, is none of them. The three components are part of the FL that characterizes us as a species; all three are the product of natural evolution and, therefore, do not vary in historical time. None of them has changed substantially in the last 1,500 years, although in that time we have gone from Latin to Spanish, two very different languages.

To understand this answer, we need to dig a little deeper into how these three essential ingredients relate to each other. Some authors have proposed that the relationship between the computational system and the conceptual and sensorimotor systems is asymmetric, in the sense that the computational system, or syntax, would have evolved adapting to the conceptual system, forming an internal language of thought (IloT) aimed at the creation of thought. ${ }^{10}$

That IloT, essentially common to the species, and probably the hallmark of human cognition, would later have been connected to the sensorimotor system for externalization and, therefore, for communication, probably reusing an old communication system. According to this vision, externalization would be secondary, that is, a process exposed to fluctuation in the environment and therefore susceptible to change and diversification.

This scenario implies that any I-language must also include a component derived from the environment-that is, internalized-whose mission would be to systematically connect the structures generated by the IloT with the sensorimotor system. The crucial idea now is that this component is the only one that results from learning and therefore is also the only one that is exposed to change and variation. Let us call this component the internalized-lexicon, or I-lexicon.

Note that it is then implied that the essential function of language-understood as semantics + syntax-would be thought, not communication. Although very important for our species, communication is a secondary use of language. ${ }^{11}$

The connection of the ILoT with the sensorimotor system then allows the externalization of thoughts as physical signals, typically sound waves, that can go from one individual to another. But for this to be possible, it is necessary to establish a shared link between, on the one hand, abstract syntactic-semantic representations and, on the other, the system capable of producing signals through muscle movements. That is the function of the I-lexicon, a domain of long-term memory that provides a stable and I-language-specific connection between the internal syntactic-semantic structures and the sensorimotor systems that process and produce the signals that human beings perceive and produce when they use language for communication.

The I-lexicon can be thought of as a repertoire of exponents, or morphemes, that systematically match abstract syntactic structures and sounds, that is, that translate the former into the latter. ${ }^{12}$ As these exponents can be different in each language, we can say that the I-lexicon-the code to link thoughts and sounds-is responsible for the diversity of languages. The reason that the exponents we use to externalize language may be different in different speech communities is that those exponents are cultural entities passed down from generation to generation.

According to this model, learning the language of the environment actually involves the task of learning to externalize the ILoT in the same way that other members of the linguistic community do. In this process, mismatches between form and structure, which are traditionally known in historical linguistics as reanalyses, can occur. A reanalysis is an alteration of the relationship between an underlying structure and a linguistic expression. These relations are the only thing that linguistic changes can alter.

If this is so, the claim that language change would be responsible for language evolution is meaningless, since language change cannot in any way alter the components of the FL that have not been learned.

\section{How and Why Language Change Takes Place}

Although I have argued that language change and language evolution are different processes, it is still true, as Darwin himself suggested, that the mechanisms of linguistic and biological change are similar. Thus, I suggest that the reanalysis mechanism in language change is the equivalent to the genetic mutation in natural evolution.

Consider a simplified example, such as the passage from the analytical late Latin future-amare habeo, lit. "(I) to love have," "I will love"-to the synthetic Romance future-e.g., Spanish amaré, "I will love." The essential idea is that for speaker $\mathrm{S}_{1}$ expression $\mathrm{E}$ /amaré/ has a specific structure-roughly amar $+\dot{e}=\mathrm{V}+$ Aux.Tense-while for speaker $\mathrm{S}_{2}$ the same expression $\mathrm{E}$ has a different structure-amaré = V.Tense. Speaker $S_{2}$ reanalyses expression $\mathrm{E}$ and assigns it a different underlying structure than speaker $\mathrm{S}_{1}$. The I-language, or the I-lexicon, of speaker $\mathrm{S}_{2}$ then has a mutation, in the sense that the relation between the expression $\mathrm{E}$ and the elements of its underlying struc- 
ture is different from that of the I-language of speaker $\mathrm{S}_{1}$.

The task of listeners is to use their I-language, including their own I-lexicon, to discover meaning by analysing the sound wave. In the ideal case, the syntactic-semantic structure that a listener obtains is identical to the one that the speaker had in mind. When this is not the case, we can say that a reanalysis has occurred. A reanalysis is basically a decoding or acquisition error. When that error or mutation stabilizes in the listener's I-language and spreads to other speakers, we say that a linguistic change has occurred.

\section{Conclusion}

There is no evidence that the oldest known or reconstructed languages were more primitive, less efficient, or less sophisticated than current languages. Languages are not cultural inventions, but a complex mix of biology and culture. The parts of languages subject to cultural transmission and change are concentrated in the I-lexicon. There is no reason to think that this type of cultural change has been the causal factor in the emergence of the human faculty of language. For this reason, the expressions language evolution and language change should be kept separate.

José-Luis Mendivil-Giró is a Professor in the Department of General and Hispanic Linguistics at the University of Zaragoza.

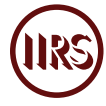

1. James Hurford, "An Approach to the Phylogeny of the Language Faculty," in The Evolution of Human Languages, ed. John Hawkins and Murray Gell-Mann (Reading, MA: Addison-Wesley, 1992): 273.

2. For example: John Batali, "Computational Simulations of the Emergence of Grammar," in Approaches to the Evolution of Language: Social and Cognitive Bases, ed. James Hurford, Michael Studdert-Kennedy, and Chris Knight (Cambridge: Cambridge University Press, 1998), 405-26; Simon Kirby, "Fitness and the Selective Adaptation of Language," in Approaches to the Evolution of Language, 359-83; Simon Kirby, "Syntax without Natural Selection: How Compositionality Emerges from Vocabulary in a Population of Learners," in The Evolutionary Emergence of Language: Social Function and the Origins of Linguistic Form, ed. Chris Knight, Michael Studdert-Kennedy, and James Hurford (Cambridge: Cambridge University Press, 2000), 303-23, doi:10.1017/CBO9780511606441.019; Simon Kirby, "Learning, Bottlenecks and the Evolution of Recursive Syntax," in Linguistic Evolution through Language Acquisition: Formal and Computational Models, ed. Ted Briscoe (Cambridge:
Cambridge University Press, 2002), 173-203, doi:10.1017/ CBO9780511486524.006; Luc Steels, "Synthesizing the Origins of Language and Meaning Using Coevolution, Self-Organization and Level Formation," in Approaches to the Evolution of Language, 384-404; Bernd Heine and Tania Kuteva, The Genesis of Grammar (Oxford: Oxford University Press, 2007); Thomas Givon, The Genesis of Syntactic Complexity (Amsterdam and Philadelphia: John Benjamins, 2009), doi:10.1075/z.146; Daniel Dor and Eva Jablonka, "Plasticity and Canalization in the Evolution of Linguistic Communication: An Evolutionary Developmental Approach," in The Evolution of Human Language: Biolinguistic Perspectives, ed. Richard Larson, Viviane Déprez, and Hiroko Yamakido (Cambridge: Cambridge University Press, 2010), 135-47, doi:10.1017/CBO9780511817755.010.

3. For example: Derek Bickerton, Language and Species (Chicago: Chicago University Press, 1990); Steven Pinker and Paul Bloom, "Natural Language and Natural Selection," The Behavioral and Brain Sciences 13 (1990): 707-84, doi:10.1017/ S0140525X00081061; Noam Chomsky, "Some Simple Evo Devo Theses: How True Might They Be for Language?" in The Evolution of Human Language, 45-62, doi:10.1017/ CBO9780511817755.003; April McMahon and Robert McMahon, Evolutionary Linguistics (Cambridge: Cambridge University Press, 2013); Robert Berwick and Noam Chomsky, Why Only Us: Language and Evolution (Cambridge: MIT Press, 2016).

4. I have made this argument in more detail in José-Luis Mendívil-Giró, "Did Language Evolve through Language Change? On Language Change, Language Evolution and Grammaticalization Theory," Glossa: A Journal of General Linguistics 4, no. 1 (2019): 124, doi:10.5334/gjgl.895.

5. Östen Dahl, The Growth and Maintenance of Linguistic Complexity (Amsterdam and Philadelphia: John Benjamins, 2004), doi:10.1075/slcs.71.

6. Chomsky, "Some Simple Evo Devo Theses."

7. Samuel Epstein, Hisatsugu Kitahara, and T. Daniel Seely, "Derivation(s)," in Explorations in Maximizing Syntactic Minimization, ed. Epstein, Kitahara, and Seely (New York: Routledge, 2015), 1.

8. Sign languages are based on visual and non-auditory stimuli, but the underlying logic is the same, as it is the same language faculty that deaf people use to learn and use these languages.

9. Marc Hauser, Noam Chomsky, and W. Tecumseh Fitch, "The Faculty of Language: What Is It, Who Has It, and How Did It Evolve?" Science 298, no. 5,598 (2002): 1,569-79, doi:10.1126/ science.298.5598.1569.

10. Berwick and Chomsky, Why Only Us.

11. Another serious terminological problem, worthy of being on Leivada's list, is that Chomsky uses the term "language" for the syntax + semantics system, while other authors use it only for the externalization component. See Mendívil-Giró, "Did Language Evolve through Language Change?" for discussion. 
12. According to the nanosyntax model, syntactic heads are typically submorphemic, so each lexical entry of the I-lexicon matches a fragment of a syntactic tree with a phonological representation. See Michal Starke, "Nanosyntax: A Short Primer to a New Approach to Language," Nordlyd 36, no. 1 (2010): 1-6, doi:10.7557/12.213.

Published on September 28, 2020 